\title{
Avaliação do trabalho em equipe na Unidade de Terapia Intensiva Materna com relação a Cultura de Segurança do Paciente
}

\author{
Evaluation of teamwork in the Maternal Intensive Care Unit in relation to the Culture of Patient \\ Safety \\ Evaluación del trabajo en equipo en la Unidad de Cuidados Intensivos Maternos en relación a la \\ Cultura de Seguridad del Paciente
}

José Augustinho Mendes Santos ORCID: https://orcid.org/0000-0002-1570-4102 Universidade Federal de Alagoas, Brasil E-mail: augustinhomendes1@gmail.com

Amuzza Aylla Pereira dos Santos ORCID: https://orcid.org/0000-0001-6299-7190 Universidade Federal de Alagoas, Brasil E-mail: amuzza.santos@gmail.com

Thaís Honório Lins Bernardo

ORCID: https://orcid.org/0000-0002-8058-8400 Universidade Federal de Alagoas, Brasil E-mail: thais.bernardo@eenf.ufal.br Mari Ângela Gaedke

ORCID: https://orcid.org/0000-0002-3032-081X Universidade de Santa Cruz do Sul, Brasil E-mail: marig@unisc.br

Isabel Comassetto

ORCID: https://orcid.org/0000-0002-2389-9384 Universidade Federal de Alagoas, Brasil

E-mail: isabelcomassetto@gmail.com

Fernanda dos Santos Iochims

ORCID: https://orcid.org/0000-0001-9065-6561 Universidade de Santa Cruz do Sul, Brasil

E-mail: fernanda.iochims@gmail.com

Gilmara Salustiano Santos

ORCID: https://orcid.org/0000-0002-3795-0590 Universidade de Ciências da Saúde de Alagoas, Brasil

E-mail: gil.salustiano@hotmail.com

\begin{abstract}
Resumo
O objetivo deste estudo foi avaliar o trabalho em equipe de uma unidade de terapia intensiva materna, na perspectiva da equipe multiprofissional de saúde com relação a cultura de segurança do paciente. Estudo transversal, realizado entre fevereiro e março de 2021, que utilizou para a coleta de dados o questionário Hospital Survey on Patient Safety Culture, que avalia 12 dimensões da Cultura de Segurança do Paciente sendo para fins deste estudo, avaliado os itens que compõem as dimensões "Trabalho em equipe dentro da unidade" e "Trabalho em equipe entre as unidades", constituindo assim, 8 itens. Participaram do estudo 40 profissionais. Ao analisar os itens avaliados, observou-se que 3 foram considerados áreas de força para a CSP, 1 como área neutra e 4 classificados como frágeis. Pode-se afirmar que na perspectiva da equipe multiprofissional da UTIM, o trabalho em equipe dentro da unidade é forte para a CSP, pois eles se tratam com respeito, apoiando uns aos outros, além de trabalharem como equipe quando há muito trabalho a ser realizado. No que se refere ao trabalho em equipe entre as unidades, os profissionais acreditam que os setores das maternidades não estão bem coordenadas entre si e que não existe uma boa cooperação entre as unidades que precisam trabalhar em conjunto.
\end{abstract}

Palavras-chave: Qualidade da assistência à saúde; Segurança do paciente; Comunicação em saúde; Equipe de assistência ao paciente; Unidades de Terapia Intensiva.

\section{Abstract}

The objective of this study was to evaluate the teamwork in a Maternal Intensive Care Unit (MICU), from the perspective of the multiprofessional health team. This is a cross-sectional study, conducted between February and March 2021, which used for data collection the Hospital Survey on Patient Safety Culture questionnaire, which assesses 12 dimensions of Patient Safety Culture (CSP). For the purposes of this study, the items that make up the 
dimensions "Teamwork within the unit" and "Teamwork between units" were assessed, thus constituting 8 items. Forty professionals participated in the study. When analyzing the items evaluated, it was observed that 3 were considered areas of strength for the CSP, 1 as a neutral area and 4 classifieds as weak. It can be stated that from the perspective of the multiprofessional team of the UTIM, teamwork within the unit is strong for the CSP, because they treat each other with respect, supporting each other, besides working as a team when there is a lot of work to be done. Regarding teamwork between the units, the professionals believe that the sectors of the maternity hospitals are not well coordinated among themselves and that there is not good cooperation between the units that need to work together.

Keywords: Quality of health care; Patient safety; Health communication; Patient care team; Intensive Care Units.

\section{Resumen}

El objetivo de este estudio fue evaluar el trabajo en equipo en una Unidad de Cuidados Intensivos Maternales (UCIM), desde la perspectiva del equipo sanitario multiprofesional. Estudio transversal realizado entre febrero y marzo de 2021, en el que se utilizó para la recogida de datos el cuestionario de la Encuesta Hospitalaria sobre Cultura de Seguridad del Paciente, que evalúa 12 dimensiones de la Cultura de Seguridad del Paciente (CSP), siendo para los fines de este estudio, evaluados los ítems que conforman las dimensiones "Trabajo en equipo dentro de la unidad" y "Trabajo en equipo entre unidades", constituyendo así 8 ítems. Cuarenta profesionales participaron en el estudio. Al analizar los ítems evaluados, se observó que 3 fueron considerados como áreas de fortaleza para el CSP, 1 como área neutral y 4 clasificados como frágiles. Se puede afirmar que desde la perspectiva del equipo multiprofesional de la UTIM, el trabajo en equipo dentro de la unidad es fuerte para el CSP, porque se tratan con respeto, apoyándose mutuamente, además de trabajar en equipo cuando hay mucho trabajo por hacer. En cuanto al trabajo en equipo entre las unidades, los profesionales creen que los sectores de las maternidades no están bien coordinados entre sí y que no hay una buena cooperación entre las unidades que deben trabajar juntas.

Palabras clave: Calidad de la atención sanitaria; Seguridad del paciente; Comunicación sanitaria; Equipo de atención al paciente; Unidades de Cuidados Intensivos.

\section{Introdução}

Nos últimos anos, os movimentos criados em busca da prestação do cuidado com qualidade nos serviços de saúde, pautados na Segurança do Paciente (SP) tem se destacado a nível nacional e internacional, embora ainda não seja uma temática discutida de maneira mais ampla (Minuzzi, et al., 2016). Um dos atributos discutidos sobre a qualidade dos serviços de saúde é o trabalho em equipe, que se trata de um elemento essencial para a SP, principalmente considerando os fatores de maior complexidade que envolvem as doenças, o aumento das especializações no atendimento e das comorbidades, a escassez de força de trabalho e o incremento das inovações tecnológicas cada vez mais presentes em todos os cenários de assistência ao paciente (Bohomol, 2015).

O trabalho dentro das instituições de saúde ainda é predominantemente desarticulado, visto que cada grupo profissional planeja, executa e avalia suas ações em geral de forma isolada, sem articulação interdisciplinar, que poderia potencializar amplamente a integralidade das ações (Lima et al., 2018).

Nesta perspectiva, percebe-se que a equipe multiprofissional desrespeita a complexidade existencial do indivíduo, da família e comunidades, produzindo um trabalho fragmentado, que por vezes não atende as demandas e necessidades em saúde em diferentes dimensões. Assim, uma boa integração dentre os atores profissionais reflete diretamente na qualidade do cuidado ofertado ao cliente, e consolidam um novo formato organizativo da unidade de saúde. Para que isto seja possível, é imprescindível conceber o cuidado integral desde a organização do serviço, formação profissional até alcançar a atuação assistencial, para então se ter um trabalho em equipe (Silva, et al., 2018).

O trabalho em equipe, trata-se de uma ferramenta do processo de trabalho em saúde que, para seu pleno funcionamento, requer de todos os membros da equipe a composição de um conjunto de instrumentos para construção e consolidação de espaços de troca entre os profissionais, estimulando os vínculos entre estes (Laccort \& Oliveira, 2017). Esta compreensão permite visualizar como a harmonia da equipe se converte em estratégia para abordagem terapêutica assertiva, ampliada e contextualizada, capaz de prevenir, promover e tratar problemas de saúde (Peduzzi, et al., 2020). 
As competências relativas à comunicação interprofissional, objetivos comuns, reconhecimento do trabalho dos demais componentes da equipe, interdependência das ações, colaboração interprofissional e atenção centrada no usuário, surgem precipuamente ao cuidado integral e humanizado. Assim, quanto mais dialógicas forem as relações de trabalho, mais coesas serão as intervenções e planejamentos de cuidado à saúde, caso contrário, não haverá contribuições multiprofissionais integradas, produzindo uma dinâmica de trabalho fragmentado, e por conseguintes ações menos resolutivas (Peduzzi, et al., 2020).

Assim, o trabalho em equipe implica em relações e noções de trabalho coletivas, baseadas em reciprocidade e complementaridade do pensar perito de cada profissional, para conceber e resolver a complexidade das necessidades humanas do cliente (Navarro et al., 2013). O relacionamento entre as pessoas proporciona um ambiente agradável e harmonioso, características que contribuem diretamente para o desenvolvimento de um bom trabalho em equipe, bem como na qualidade dos serviços prestados (Laccort \& Oliveira, 2017).

Atualmente, qualidade e resultados positivos no setor de saúde são exigências na gestão de processos de trabalhos coletivos. Considerando a complexidade das unidades que compõem uma instituição de saúde, direcionamos neste estudo especial atenção à Unidade de Terapia Intensiva (UTI), que se caracteriza como um setor fechado, com estrutura e dinâmica próprias voltadas à promoção do cuidado. Em consonância com as demandas e fragilidades do perfil de pacientes que esta unidade acolhe, o setor é equipado com vasto aparato tecnológico, altamente instrumentalizado e práticas racionalizadas. Os cuidados críticos são construídos a partir da intervenção colaborativa, onde diferentes categorias apresentam suas contribuições especializadas para intervenção junto ao paciente. Com isso, se tem um cuidado que acumula diversas perspectivas e abordagens adequadamente conectadas para um mesmo fim, o cuidado integral e holístico do paciente crítico (Nogueira \& Rodrigues, 2015; Caram, et al., 2016).

$\mathrm{O}$ trabalho em UTI exige ainda novas competências dos profissionais, que se deparam com mudanças tecnológicas e exigências de sua clientela, provocando muitas vezes transformações no seu processo de trabalho. Na UTI, a estratégia do trabalho em equipe torna-se proposta que enfatiza a cooperação e incorpora a participação dos profissionais, fortalecendo o trabalho multiprofissional e gerando repercussões diretas na assistência integral prestada, tornando-se, assim, estratégia primordial para a gestão das pessoas (Camelo \& Chaves, 2013).

No que se refere ao trabalho em equipe no âmbito obstétrico, percebe-se que o desempenho das equipes representa um componente essencial para a eficiência clínica de equipes multiprofissionais, já que está intimamente relacionada à qualidade dos serviços ofertados e com a SP (Fransen, et al., 2017). A assistência à mulher no ciclo gravídico-puerperal não deve ser disputada e sim compartilhada entre os profissionais da saúde, para que eles planejem como o cuidado deve ser executado planejamento este de suma importância para que se garanta uma assistência de qualidade às gestantes e puérperas (Lima et al., 2018).

A interface clínico-obstétrica assume especial importância em se tratando de uma Unidade de Cuidados Intensivos para mulheres no ciclo grávido-puerperal. As alterações fisiológicas características da gestação distinguem essas pacientes de outros adultos jovens. As afecções obstétricas diretas e indiretas podem levar mulheres que vivem o ciclo gravídico puerperal a serem admitidas em uma UTI, considerando a gestação como um período de intensas modificações orgânicas que exigem uma maior atenção e assistência especializada. Entende-se, aqui, a importância da Unidade de Terapia Intensiva (UTI) exclusiva ao atendimento da mulher no ciclo gravídico-puerperal (Araújo et al., 2018; Mourão, et al., 2019).

Destaca-se ainda que o trabalho em equipe nas unidades e entre as unidades que compõem uma instituição de saúde são dimensões importantes da Cultura de Segurança do Paciente (CSP) e se faz necessário que estas dimensões estejam fortalecidas para que seja possível prestar uma assistência de qualidade. O foco central do cuidado das equipes de saúde deve 
estar direcionado sempre ao paciente, garantindo aos mesmos o direito de um atendimento que minimize a incidência de eventos adversos decorrentes de déficits no trabalho em equipe.

Diante do exposto, emergiu a seguinte questão norteadora para o estudo: como acontece o trabalho em equipe dos profissionais multiprofissional de uma Unidade de Terapia Intensiva Materna (UTIM) com relação a cultura de segurança do paciente?

Para responder à pergunta norteadora, elaborou-se o seguinte objetivo: avaliar o trabalho em equipe de uma unidade de terapia intensiva materna, na perspectiva da equipe multiprofissional de saúde com relação a cultura de segurança do paciente.

\section{Metodologia}

Trata-se de um estudo do tipo transversal, realizado em uma Unidade de Terapia Intensiva Materna (UTIM) de um estado de região Nordeste do Brasil. Destaca-se que as pesquisas transversais envolvem a coleta de dados em um determinado ponto do tempo, com o objetivo de observar e explorar os diversos aspectos de uma situação (Polit \& Beck, 2011).

A população de estudo foi constituída por 40 profissionais da equipe multiprofissional de saúde. A amostra foi intencional, não probabilística, sendo utilizados os seguintes critérios de inclusão: profissionais de ambos os sexos, que estivessem no exercício de suas funções na UTIM no período de coleta de dados há pelo menos 6 meses, que prestassem assistência direta aos pacientes e que possuíssem carga horária de trabalho de 12 horas semanais, devido a escalas fracionadas. Foram excluídos do estudo os profissionais que estivessem afastados devido a licença saúde, maternidade, e aquelas que foram afastados devidos a decretos publicados como medidas de prevenção conta a COVID-19, além daqueles que após 3 tentativas não foram encontrados na unidade de trabalho.

A coleta de dados ocorreu entre fevereiro e março de 2021 e deu-se por meio da aplicação do instrumento Hospital Survey on Patient Safety Culture (HSOPSC), que é de origem norte-americana, mas que foi traduzido e validado para o contexto hospitalar brasileiro (Reis et al., 2016). Os profissionais foram abordados em seu ambiente de trabalho, e após explicação dos objetivos do estudo e forma de participação eles foram convidados a participar do estudo. Após manifestarem aceite em participar, foi entregue duas vias do Termo de Consentimento Livre e Esclarecido e uma via do instrumento de coleta de dados em um envelope contendo o instrumento que foi preenchido individualmente pelos participantes e após inserido no envelope e grampeado.

O HSOPSC avalia 12 dimensões da cultura de segurança do paciente, além de possuir itens que avaliam o perfil sociodemográfico e de trabalho. Na busca de responder a questão norteadora deste estudo, bem como pelo atendimento integral do objetivo desta investigação, optou-se por analisar as dimensões "Trabalho em equipe na unidade" e "Trabalho em equipe entre as unidades", por considerar que estas dimensões melhor retratam a cultura de segurança do paciente e a sua interface com o trabalho em equipe, totalizando 8 itens avaliados, 4 para cada dimensão. Ambos os itens são respondidos através de uma escala do tipo Likert, graduada em cinco pontos, que refletem o grau de concordância, de 1 (discordo totalmente) a 5 (concordo totalmente).

Após a coleta de dados, os instrumentos preenchidos passaram por uma dupla avaliação exploratória para verificar a existência de respostas altamente assimétricas, em seguida eles foram inseridos em uma planilha em formato Microsoft Excel, por dupla digitação e validados. Posteriormente foram transferidos para o software estatístico JASP, versão 0.16, e analisados através da estatística descritiva, através de frequência absoluta e relativa, além de medidas de dispersão para algumas variáveis sociodemográficas, como média e desvio padrão.

A avaliação dos itens foi realizada por meio do percentual de respostas negativas, neutras e positivas, obtidas em cada um dos mesmos. As respostas foram então classificadas em: respostas positivas: respostas onde foram assinaladas as opções 4 
ou 5 (concordo/concordo totalmente) para itens formulados de forma positiva em relação à segurança do paciente, ou as opções 1 ou 2 (discordo/discordo totalmente) nas perguntas formuladas negativamente.; respostas neutras: respostas onde foram assinaladas a opção 3 (nem concordo, nem discordo) para qualquer pergunta; respostas negativas: respostas onde foram assinaladas as opções 1 ou 2 (discordo/discordo totalmente) para itens formulados de forma positiva em relação à segurança do paciente, ou as opções 4 ou 5 (concordo/concordo totalmente) nas perguntas formuladas negativamente.

Para avaliar as dimensões foram identificadas áreas frágeis e fortes da cultura de segurança do paciente. São considerados áreas fortes as dimensões cujos itens atingiram 75\% de respostas positivas, áreas neutras são aquelas onde itens apresentaram percentuais de respostas positivas acima 50\% e inferiores a 75\%. e áreas frágeis cujos itens receberam menos de 50\% de respostas positivas (Sorra et al., 2018).

As questões éticas seguiram as recomendações das Resoluções 466/2012 e 510/2016 do Conselho Nacional de Saúde, sendo o projeto de pesquisa submetido na Plataforma Brasil e aprovado pelo Comitê de Ética em Pesquisa com Seres Humanos da Universidade Federal de Alagoas-UFAL, sob parecer nº 4.297.880.

\section{Resultados}

Aceitaram participar deste estudo 40 profissionais que compõem o quadro de funcionários da UTIM, representando um percentual de 83,3\%, divididos entre enfermeiros, auxiliares e técnicos de enfermagem, médicos e fisioterapeutas. No que se refere as características sociodemográficas, 37 (92,5\%) eram mulheres. No que tange a idade, encontrou-se uma média de 46,2 com um desvio padrão de 5,3 e uma idade mínima de 35 anos e máxima de 57 anos. Ao analisar a escolaridade, observouse que, $75 \%$ possuíam pós-graduação.

Observou-se que ao analisar o tempo de trabalho na maternidade, 75,0\% têm entre 10 e 20 anos de trabalho. Já com relação ao tempo de trabalho UTIM, 52\% relataram possuir entre 16 a 20 anos 17,5\% entre 11 e 15 anos de experiência profissional. No que concerne a carga horária de trabalho na UTIM, 87,5\% afirmaram trabalhar entre 20 a 39 horas semanalmente, em escala de plantão de 12 horas por 36 horas de descanso. Em relação ao tempo de trabalho na profissão, encontrou-se uma média de 19,3 anos, com um desvio padrão de 6,7 e com mínima e máxima de 6 e 31 anos respectivamente. especialidade $47,5 \%$ possuem entre 16 e 20 anos, seguido de $35 \%$ com 21 anos ou mais.

Ao avaliar o trabalho em equipe no contexto da UTIM e entre as unidades com foco na CSP a partir dos 8 itens avaliados, pode-se perceber, conforme os dados apresentados na tabela 1, que 3 itens é considerado forte para a CSP, pois receberam, percentuais de respostas positivas superior a 75\%, 1 é considerado neutro e 4 na perspectiva da equipe da UTIM é considerado frágil, pois receberam percentuais de respostas positivas inferior a $50 \%$.

O item com maior percentual de respostas positivas foi "Nesta unidade, as pessoas se tratam com respeito", com um percentual de $97,5 \%$, destaca-se ainda que este item não recebeu respostas negativas. Em relação ao item com o menor percentual de resposta positivas foi o "Há uma boa cooperação entre as unidades do hospital que precisam trabalhar em conjunto", com um percentual de 17,5\%, este mesmo item recebeu o maior percentual de respostas neutras entre os 8 itens avaliados. Quando analisado o item que recebeu o maior percentual de respostas negativas, tem-se o item "As unidades do hospital não estão bem coordenadas entre si”, com 50\% de resposta negativa (Tabela 1). 
Tabela 1: Distribuição das respostas negativas, neutras e positivas dos itens relacionas ao trabalho em equipe, na perspectiva de profissionais de uma Unidade de Terapia Intensiva Materna. Brasil, 2021.

\begin{tabular}{lccc}
\hline \multicolumn{1}{c}{ Dimensão } & $\begin{array}{c}\text { Negativas } \\
\mathbf{n}(\%)\end{array}$ & $\begin{array}{c}\text { Neutras } \\
\mathbf{n}(\%)\end{array}$ & $\begin{array}{c}\text { Positivas } \\
\mathbf{n}(\%)\end{array}$ \\
\hline Nesta unidade, as pessoas apoiam umas às outras. & $05(12,5)$ & $03(7,5)$ & $32(80,0)$ \\
\hline $\begin{array}{l}\text { Quando há muito trabalho a ser feito rapidamente, trabalhamos juntos em } \\
\text { equipe para conclui-lo devidamente. }\end{array}$ & $03(7,5)$ & $03(7,5)$ & $34(85,0)$ \\
\hline Nesta unidade, as pessoas se tratam com respeito. & $00(0,0)$ & $01(2,5)$ & $39(97,5)$ \\
\hline $\begin{array}{l}\text { Quando uma área desta unidade fica sobrecarregada, os outros profissionais } \\
\text { desta unidade ajudam. }\end{array}$ & $11(27,5)$ & $05(12,5)$ & $24(60,0)$ \\
\hline As unidades do hospital não estão bem coordenadas entre si. & $20(50,0)$ & $08(20,0)$ & $12(30,0)$ \\
\hline $\begin{array}{l}\text { Há uma boa cooperação entre as unidades do hospital que precisam trabalhar } \\
\text { em conjunto. }\end{array}$ & $19(47,5)$ & $14(35,0)$ & $07(17,5)$ \\
\hline $\begin{array}{l}\text { Muitas vezes e desagradável trabalhar com profissionais de outras unidades do } \\
\text { hospital. }\end{array}$ & $15(37,5)$ & $07(17,5)$ & $18(45,0)$ \\
\hline $\begin{array}{l}\text { As unidades do hospital trabalham bem em conjunto para prestar o melhor } \\
\text { cuidado aos pacientes. }\end{array}$ & $17(42,5)$ & $13(32,5)$ & $10(25,0)$ \\
\hline
\end{tabular}

Fonte: Dados da pesquisa (2021).

\section{Discussão}

Os profissionais que participaram do estudo nas maternidades pesquisadas apresentavam idade média de 46,2 anos. Tais resultados se aproximam dos encontrados por Cavalcante (2017) em estudo realizado em hospital de ensino do Piauí, com idade média de 45,5 anos. Tal resultado pode estar associado ao fato de que as instituições de saúde a qual este estudo foi realizado pertencente a instituições públicas, o que garante estabilidade no trabalho, visto que a maioria dos profissionais atuam em caráter estatuário.

Observou-se que a maioria dos participantes possuem pós-graduação, corroborando com os achados do estudo de Carmo, et al. (2020). Este resultado pode estar associado, pelo fato de as instituições pesquisadas serem maternidades de ensino ligadas a Universidades, além disso pelo crescente aumento da procura por cursos de graduação e pós-graduação, afins de melhor posicionamento no mercado de trabalho. Pôde-se observar que a maioria dos profissionais pertencia à equipe de enfermagem, notadamente pela categoria de técnicos e/ou auxiliares de enfermagem. Esse perfil é compatível com a realidade local, na qual o maior contingente de trabalhadores de enfermagem nos hospitais é composto por auxiliares e técnicos de enfermagem. Estudos sobre SP realizados no Brasil, em diversos cenários no âmbito hospitalar, mediante a aplicação do HSOPSC, também apontam essa realidade no que se refere ao percentual de trabalhadores de enfermagem (Galvão, et al., 2018; Santos, et al., 2019; serrano, et al., 2019; Notaro, et al., 2019).

No que se refere as características profissionais dos participantes deste estudo, os resultados apontam que os profissionais têm experiência profissional e tempo de serviço, o que possibilita conhecer a CSP nas maternidades e de sua unidade de atuação, além de trabalharem em uma jornada de trabalho conforme legislações trabalhistas vigentes. Sabe-se que, quando a jornada de trabalho é excessiva, o cuidado não é prestado com qualidade, o que pode gerar EA e afetar a SP (Tst, 2021).

Quando analisado os itens avaliados, percebe-se que dos oito, observa-se três que são fortes para a CSP e um é considerado neutro, com potencial de melhorias. Percebe-se ainda que estes itens são relacionados a unidade a qual os profissionais estão atuando, ou seja, na UTIM. No que se refere as fragilidades para a CSP, tem-se quatro itens, e nota-se que são aqueles que estão interligados com o trabalho em equipe entre as unidades da maternidade.

No que se refere ao item "Nesta unidade, as pessoas apoiam umas às outras", foi considerada forte para a CSP, denotando que na UTIM, os profissionais se apoiam, o que é considerado muito importante, pois a interação da equipe favorece o envolvimento e o comprometimento, fazendo que os profissionais realizem seu trabalho com mais prazer, tendo 
como consequência a qualidade do atendimento prestado. Além disso, quando o profissional não consegue se afeiçoar as atividades do trabalho, a realização das tarefas pode causar frustrações, fazendo com que ele se sinta desconfortável, além disso, a convivência na unidade de atuação, torna-se insatisfatória e a falta de reconhecimento provoca sentimento de inferioridade, causando impotência e baixa autoestima. Nesse direcionamento, os profissionais lançam mão de estratégias de defesa, buscando significar as tarefas que precisam ser realizadas (Caram, et al., 2016).

$\mathrm{O}$ item "Quando há muito trabalho a ser feito rapidamente, trabalhamos juntos em equipe para conclui-lo devidamente", recebeu $85 \%$ de respostas positivas, o que infere ser uma área forte dentro da UTIM. Tal resultado corrobora com os achados de um estudo realizado em uma maternidade pública do estado do Ceará (Barrilas, et al., 2021). Em relação ao item "Nesta unidade, as pessoas se tratam com respeito", foi a que recebeu o melhor percentual de respostas positivas, com um percentual de quase $100 \%$, corroborando com um estudo realizado em um centro de referências em cardiopeneumologia, considerando no contexto de avalição as unidades de cuidado críticos, denotando ser um item positivo em outros áreas de atuação (Ribeiro, et al., 2019). É importante ressaltar que as características e diferenças individuais de cada pessoa, tais como, o temperamento, o caráter e personalidade, podem ser consideradas possíveis impedimentos para o relacionamento interpessoal e, por conseguinte, poderão afetar na forma de desenvolvimento do trabalho na equipe (Navarro; Guimarães \& Garanhani, 2013).

Quando analisado o item "Quando uma área desta unidade fica sobrecarregada, os outros profissionais desta unidade ajudam”, observou-se que não é uma área forte para a CSP na UTIM, porém encontra-se neutra, ou seja, com potencial de melhorias, para que isto aconteça se faz necessário eliminar algumas barreiras, como o fato de que algumas categorias profissionais, por mais que estejam sobrecarregas, não aceitam ajuda, por achar que determinada conduta pode não ser realizado por outra categoria profissional. Destarte, no que concerne às barreiras ao exercício do trabalho em equipe, pode-se apontar a adoção de atitudes impróprias tais como o individualismo, falha de comunicação, desrespeito à diversidade, a antipatia e o mau humor que, prejudicam consideravelmente o desenvolvimento dos trabalhos e transformam o ambiente laboral num local desagradável e altamente contrário às práticas que visem a harmonização de ideias e ações em prol do alcance dos objetivos traçados pelas empresas (Cruz \& Braz, 2017).

O item "As unidades do hospital não estão bem coordenadas entre si”, foi considerada frágil, o que urge a necessidade de melhores abordagens com os profissionais, supervisores e lideranças sobre a importância do trabalho em equipe, pois, por mais que estejam em unidades diferentes, o profissionais precisam compreender que prestam serviço para a instituição como o todo, e que a instituição tem como objetivo prestar o melhor cuidado as pacientes, e como já é sabido, a mulher no ciclo gravido-puerperal, quando hospitalizada, irá passar por diversos cenários de assistência, desde o acolhimento com classificação de risco, passando pelo pré-parto, sala de parto, alojamento conjunto, e em casos graves UTIM.

Observou-se que o item "Há uma boa cooperação entre as unidades do hospital que precisam trabalhar em conjunto", foi o que recebeu o menor percentual de respostas positivas, denotando na perspectiva da equipe de profissionais da UTIM, a existência de conflitos e falta de interação entre os profissionais das unidades. Isto evidencia que a equipe precisa melhorar no sentido do desenvolvimento de habilidades e competências de socialização e comunicação dentro das relações de trabalho, que vão desde a inteligência emocional até os conhecimentos e vivências da dinâmica interpessoal e intergrupal. Ou seja, os comportamentos e atitudes dentro do relacionamento da equipe reflete a identidade daquele coletivo, que, contudo, não é rígida, sendo possível aprender novas ações e implementar aprimoramentos (Navarro; Guimarães \& Garanhani, 2013).

No que se refere ao item "As unidades do hospital trabalham bem em conjunto para prestar o melhor cuidado aos pacientes", recebeu um percentual de respostas positivas inferior e infere-se que os profissionais da UTIM, acreditam que as unidades da maternidade não estão ofertando um cuidado em conjunto, diante disto, percebe-se que o cuidado as gestantes, puérperas e recém-nascidos encontra-se comprometido, pois existe a possibilidade de não estarem ofertando um cuidado 
qualificado. Nesse sentido, é essencial promover uma boa interação entre os diferentes setores do hospital, com vistas à prestação de uma assistência segura e de qualidade ao paciente (Minuzzi, et al., 2016). Preconiza-se que exista um compromisso e empenho de todos da equipe, principalmente das lideranças em exercer uma medida de segurança do paciente efetiva, em busca de protocolos que contribuem e somem com a assistência prestada (Santos, et al., 2021).

Desde o início do século XXI, o trabalho em equipe tem sido tratado de forma associada à prática colaborativa. Esta colaboração, todavia, não deve mostrar-se circunscrita apenas às imediações de um setor ou unidade de saúde, pois o cuidado continuado e integral estende-se por vários níveis de atenção à saúde, bem como diversos setores especializados e profissionais peritos. Desta maneira, a coesão da equipe é a premissa mais importante para o cuidado integral, mas não omite a importância da comunicação com profissionais de outros serviços e setores, garantindo a continuidade do cuidado integral e coeso (Peduzzi \& Agrelli, 2018).

Em contrapartida, como benefícios trazidos pelo trabalho em equipe, destacam-se a inovação, o compartilhamento de ideias e conhecimentos entre as pessoas, a criação de novas oportunidades de aprendizagem, melhorias das relações interpessoais, expansão de competências individuais e coletivas, melhor desempenho, resolução de problemas com maior criatividade e celeridade, além de disposição para reagir às mudanças e assumir riscos (Cruz \& Braz, 2017).

Apesar das discussões e posições teóricas sobre o trabalho em equipe, ainda hoje, não se encontra esse processo de trabalho em todos os serviços de saúde. Desse modo, trabalhar em equipe não é uma das tarefas mais simples, além dos conflitos encontrados há muitas diferenças de opinião entre os profissionais. O bom relacionamento entre os integrantes é indispensável, visto que trocar ideias, aceitar opiniões diversas e saber lidar com as diferenças é uma missão necessária em todo contexto da saúde (Laccort \& Oliveira, 2017). Assim, estamos diante do desafio de olhar para a questão de como o trabalho em equipe tem sido realizado dentro das instituições de saúde. É necessário parar, melhorar o que pode estar errado, na direção de evoluir para outro conceito importante, que é o de equipe de sucesso (Bohomol, 2015).

\section{Conclusão}

Evidenciou-se no estudo que na perspectiva da equipe multiprofissional da UTIM, o trabalho em equipe dentro da unidade é forte para a CSP, pois eles se tratam com respeito, apoiando uns aos outros, além de trabalharem como equipe quando há muito trabalho e que precisa ser realizado rapidamente. Além disto, no que se refere ao trabalho em equipe entre as unidades, os profissionais acreditam que os setores das maternidades não estão bem coordenadas entre si e que não existe uma boa cooperação entre as unidades que precisam trabalhar em conjunto. Relataram ainda que as unidades da maternidade não trabalham bem em conjunto para prestar um cuidado de qualidade, além de que afirmaram ser desagradável trabalhar com profissionais de outras unidades, o que vai de encontro com a necessidade da cooperação entre as unidades.

Espera-se que os resultados deste estudo possam subsidiar estratégias de melhorias, além de uma reflexão, principalmente no que se refere ao trabalho em equipe, pois se fazem necessário que os profissionais prestem um cuidado contínuo e de qualidade para que não afete a segurança das pacientes, principalmente pela especificidade do caráter de atendimento prestados as gestantes e puérperas.

Sugere-se que outros estudos com abordagem quantitativa e qualitativa, sejam realizados para melhor compreender o trabalho em equipe em instituições de saúde, em especial em maternidades, devido ao alto número de pacientes envolvidos. Além disso, sugere-se ainda, que sejam realizados estudos que além de abordar os profissionais e gestores das instituições, também abordem os pacientes que recebem os cuidados destas equipes de saúde para fins de compreender como eles percebem o trabalho em equipe. 


\section{Referências}

Araújo, S. T., Sanches, M. E. T. L. \& Nascimento, W. S. (2018). Análise do perfil epidemiológico das internações em uma unidade de terapia intensiva materna. Enfermagem em Foco, 9 (2): 73-78.

Barillas, C. C. H., Passos, A. C. B., Júnior, C. A. A., Lopes, E. M., Neri, E. D. R, Fonteles, M. M. F. \& Santos, D. B. (2021). Cultura de segurança em uma maternidade pública de ensino: conhecer para intervir. Revista de Medicina da UFC, 61(1):1-8.

Bohomol, E. (2015). Ser participante de uma equipe eficaz. Revista da SOBECC, 20(4): 187-188.

Camelo, S. H. H. \& Chaves, L. D. P. (2013). Teamwork as a nursing competence at Intensive Care Units. Investigación y Educación en Enfermería, 31(1).

Caram, C. S., Rezende, L. C., Montenegro, L. C., Amaral, J. M. e Brito, M. J. M. (2016). Ambiguidades no trabalho da equipe de saúde no contexto de uma unidade de terapia intensiva. SANARE, 15(01): 15-24.

Cruz, M. J. E. e Braz, H. M. F. S. (2017). Trabalho em equipe: uma estratégia de gestão. Entrepreneurship, 1(1), 46-58.

Fransen, A. F., Boer, L., Kienhorst, D., Truijens, S. E., Heimel, P. J. V. R. \& Oei, S. G. (2017). Assessing teamwork performance in obstetrics: a systematic Search and review of validated tools. EJOG, 216: 184-191.

Laccort, A. A. \& Oliveira, G. B. (2017). A importância do trabalho em equipe no contexto da enfermagem. Revista UNINGÁ Review, 29(3), 6-10.

Lima, P. C., Sabino, K. C. V. \& Gouveira, M. T. O. (2018). O processo de trabalho da enfermagem obstétrica: reflexão. Enfermagem Obstétrica, 5:e30.

Malagueta, A. L. O., Silva, S. E. F. \& Figueira, C. S. A. (2019). As principais fortalezas e fragilidades na cultura de segurança do paciente: uma revisão integrativa. Saber Científico, 8(2): 156- 166.

Minuzzi, A. P., Salum, N. C., Locks, M. O. H., Amante, L. N. \& Matos, E. (2016). Contribuições da equipe de saúde visando à promoção da segurança do paciente no cuidado intensivo. Escola Anna Nery, 20(1):121-129.

Mourão, L. F., Mendes, I. C., Marques, A. D. B., Cestari, V. R. F. \& Braga, R. M. B. B. (2019). Internações em UTI por causas obstétricas. Enfermería Global, 3: 318-331.

Navarro, A. S. S., Guimarães, R. L. S. \& Garanhani, M. L. (2013). Trabalho em equipe: o significado atribuído por profissionais da estratégia de saúde da família. Revista Mineira de Enfermagem, 17(1): 61-68.

Nogueira, J. W. S. \& Rodrigues, M. C. S. (2015). Comunicação efetiva no trabalho em equipe em saúde: desafio para a segurança do paciente. Revista Cogitare Enfermagem, 20(3): 636-640.

Peduzzi, M. \& Agreli, H. L. F. (2018). Trabalho em equipe e prática colaborativa na Atenção Primária à Saúde. Interface comunicação, saúde e educação, 22(Supl. 2):1525-34.

Peduzzi, M., Agreli, H. L. F., Silva, J. A. M. \& Souza, H. S. (2020) Trabalho em equipe: uma revisita ao conceito e a seus desdobramentos no trabalho interprofissional. Trabalho, Educação e Saúde, 18(s1):e0024678.

Polit, D. F. \& Beck, C. T. (2011). Fundamentos de pesquisa em enfermagem: avaliação de evidências para a prática de enfermagem. 7. ed. Porto Alegre: Artmed, 669p.

Reis, C. T., Laguardia, J., Vasconcelos, A. G. G. \& Martins M. (2016). Confiabilidade e validade da versão brasileira do Hospital Survey on Patient Safety Culture (HSOPSC): um estudo piloto. Cadernos de Saúde Pública, 32: e00115614.

Ribeiro, A. C., Nogueira, P. C., Tronchin, D. M. R., Rossato, V. \& Serpa, L. F. (2019). Cultura de segurança do paciente: percepção dos enfermeiros em um centro de referência em cardiopneumologia. Texto \& Contexto Enfermagem, 28: e20180118.

Santos, J. A. M., Santos, A. A. P., Gaedke, M. A., Comassetto, I., Nagliate, P. C., Gallisa, F. R., Lima, E. R., Vilela, D. H. L. A., Ferro, A. P. F. \& Cunha, A. M. S. (2021). Research, Society and Development, v. 10, n. 13, e131101320898.

Santos, S. L. F., Lopes, E. M., Pereira, F. G. F. e Neri, E. D. R. (2019). Cultura de segurança do paciente: avaliação dos profissionais de saúde em unidade de terapia intensiva neonatal. EEDIC, ISSN:2446-6042.

Silva, K. R., Luiz, R. M. Q., Mello, D. S., Ribeiro, R. M., Martins, S. M., Faria, S. M. C., Fernandes, M. L., Brandão, L. G. V., Caram, C. S. \& Brito, M. J. M. (2018). Revista Médica de Minas Gerais, 28 (Supl 5): e-S280501.

Sorra J., Gray, L., \& Streagle, S. (2018). Pesquisa Hospitalar AHRQ sobre Cultura de Segurança do Paciente: Guia do Usuário. AHRQ Publication Agency for Healthcare Research and Quality., N. 18-0036-EF. 